\title{
Vibrotactile masking: Temporal integration, persistence, and strengths of representations
}

\author{
PAUL M. EVANS \\ Indiana University, Bloomington, Indiana
}

\begin{abstract}
Subjects were presented with two successive vibrotactile spatial patterns (a target and a masker) to their left index fingerpads and were instructed to identify the first pattern (Experiments 1 and 2) or the second pattern (Experiment 3). Many of the two patterns combined to form other patterns, composites, that were available to the subjects as potential responses. The temporal separation between the onsets of the two patterns (stimulus onset asynchrony, or SOA) was varied. In Experiment 1 (backward masking), subjects often erred at brief SOAs and responded with the composite of the two patterns presented. In addition, subjects sometimes responded with the masker. Both effects decreased as the SOA was increased. In Experiment 2 (backward masking), the intensity of the target was varied. As the intensity of the target was increased, the tendency to respond with the masker decreased. In Experiment 3 (forward masking), subjects again often erred at brief SOAs and responded with the composite of the two patterns presented. In addition, there was less forward masking than backward masking. Rarely, in this case, did subjects respond with the masker. It is suggested that the presentation of a vibrotactile pattern yields an internal representation that persists for some time following the cessation of stimulation. In addition, when two vibrotactile patterns are presented in close temporal and spatial proximity, they are integrated into a composite that contains a strong representation of the temporally trailing pattern.
\end{abstract}

Subjects are better able to identify a spatial, vibrotactile pattern when it is presented in isolation than when it is presented in the temporal and spatial proximity of another, different pattern (Bliss, Crane, Link, \& Townsend, 1966; Craig, 1978, 1980, 1982, 1985; Evans \& Craig, 1986). The reduction in identification performance in the presence of another pattern has been termed "masking" (Craig, 1978), and the pattern to be identified (the target) is said to be masked by the pattern to be ignored (the masker). Masking can be forward in time (when the pattern to be identified is preceded by the pattern to be ignored) or backward (when the pattern to be identified is followed by the pattern to be ignored).

Craig $(1978,1980,1982,1985)$ has investigated vibrotactile masking with letter and letter-like stimuli presented to the fingerpad using the Optacon display. The Optacon is a commercially available sensory aid for the blind that consists of a 6 column $\times 24$ row array of stimulators (Bliss, Katcher, Rogers, \& Shepard, 1970). Craig has investigated backward and forward masking, masking with patterns presented to the same site and to different sites, the effect of varying the temporal separation between the onsets of a target and masker (stimulus onset asynchrony, or SOA), and the relationship between the magnitude of the masking effect and the form of the masking stimulus.

\footnotetext{
This research was supported by Grant NS-09783 from the National Institutes of Health. The author wishes to thank James C. Craig for his comments on an earlier draft of this article, and Roger P. Rhodes for his assistance in conducting these experiments. Correspondence concerning this article should be addressed to Paul M. Evans, who is now at the Department of Psychology, Princeton University, Green Hall, Princeton, NJ 08544.
}

To summarize these findings, more backward than forward masking occurs at brief SOAs, less masking occurs when two patterns are presented to different sites than when they are presented to the same site, masking decreases as the temporal separation between the onsets of a target and masker is increased, and a pattern masker leads to significantly more masking than does an energy masker (e.g., a uniform rectangular field) when the target is a letter.

The factors underlying masking effects are still under investigation. In the visual literature, two major theoretical explanations of masking have emerged-temporal integration and interruption (Felsten \& Wasserman, 1980). According to the temporal-integration explanation, two patterns that are presented in close temporal and spatial proximity are integrated by the processing system into a composite form in which the target pattern is obscured (Averbach \& Coriell, 1961; Schultz \& Eriksen, 1977). The temporal-integration explanation can be applied to both forward- and backward-masking effects. The interruption explanation, on the other hand, applies only to backward masking. Here it is argued that the arrival of a second pattern diverts processing resources away from the target. In this case, the reduced identification performance is due to incomplete processing of the target pattern (Bongartz \& Scheerer, 1976; Spencer \& Shuntich, 1970).

Evans and Craig (1986) recently investigated the integration and interruption explanations of masking with vibrotactually presented stimuli. Using a backwardmasking paradigm, they presented subjects with target patterns that varied in the number of lines that they contained. Using the tactile array of the Optacon, the patterns were 
presented to subjects' fingerpads. When subjects misidentified a target pattern in the presence of a masker, they very often responded with a pattern that contained more lines than were contained in the target. The results of a second task, in which subjects were required to estimate the number of lines contained in a target pattern, showed that the effect of the masker was to increase the number of lines judged by subjects to be contained in a target pattern. The identification and the estimation results varied as a function of SOA. As the SOA was increased beyond $100 \mathrm{msec}$, both the pattern of identification errors and the estimations of the number of lines contained in the target pattern became similar to the results obtained in the absence of the masker. On the basis of these findings, Evans and Craig (1986) concluded that, as a result of the limited temporal resolution of the skin, two vibrotactile patterns presented in close temporal succession were integrated into a composite representation that contained more elements than were contained in the target pattern. There was no evidence that the process of interruption was contributing to the reduced identification performance.

An analysis of the confusion matrices obtained in the study by Evans and Craig (1986) suggested that particular lines (edges and contours) were being extracted from the masker and integrated with those extracted from the targets. The present study tested this notion directly. Subjects were presented with targets and maskers drawn from the same stimulus set. Many of the targets and maskers, when combined, formed other patterns, composites, that were available to the subjects as potential responses. This paradigm is similar to one used by Eriksen and Collins (1967), who presented subjects with two patterns that were meaningless by themselves but formed a letter when combined (see also Craig, 1982; Hogben \& Di Lollo, 1974). The question is, if subjects are basing their identification decisions, at brief SOAs, on a composite representation containing both the target and masker patterns, is the composite veridical with respect to the spatial locations of the lines contained in the target and masker? If it is, then we might expect subjects, at brief SOAs, not simply to err and respond with any pattern containing more lines than are contained in the target pattern, but to err and respond with a specific pattern that is the inclusive composite of the target and masker.

\section{EXPERIMENT 1}

Subjects were presented with targets and maskers that were chosen from the same stimulus set (Figure 1). Many of the pairwise combinations of these patterns form other
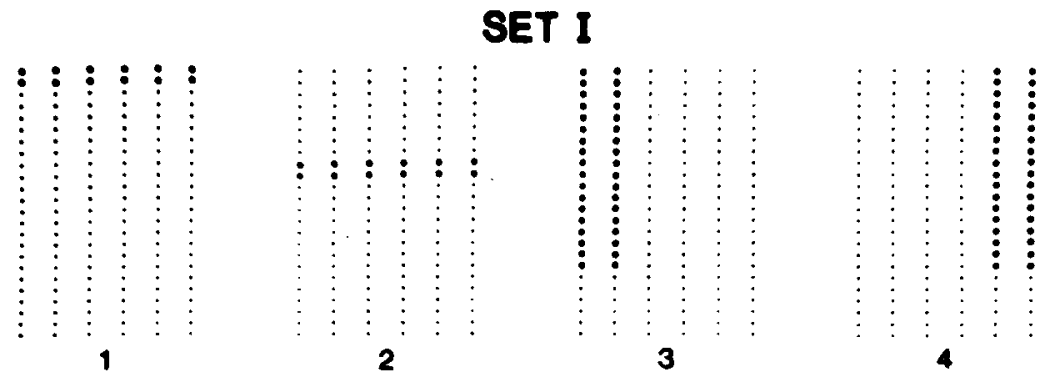
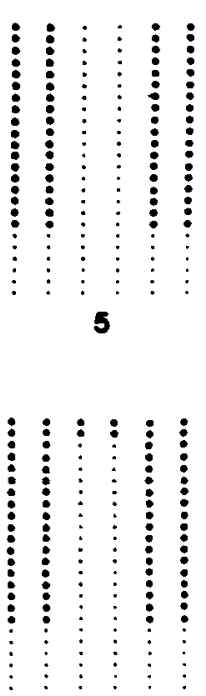

10

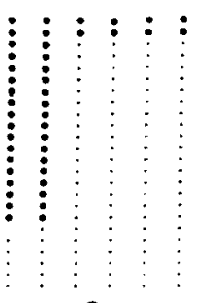

6

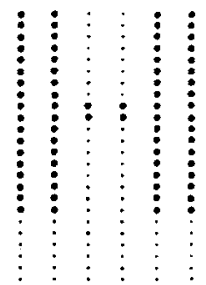

11

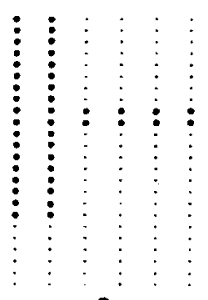

8

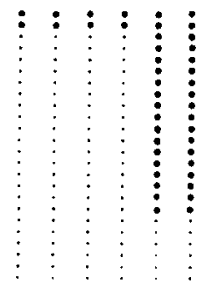

7

SET II

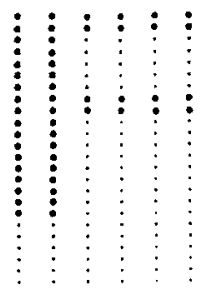

12

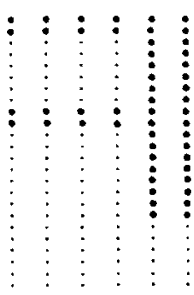

13

Figure 1. Representations of the 13 target patterns used in Experiments 1, 2, and 3. (Sets I, II, III refer to the number of lines the patterns contain, i.e., 1, 2, or 3.) These patterns were also used as the masking stimuli. 
patterns, composites, that are members of the stimulus set and thus available to subjects as responses. For example, Patterns 3 and 4 combine to form Pattern 5. If, as suggested by Evans and Craig (1986), temporal integration is a major factor involved in vibrotactile masking, and if information about the spatial locations of lines is preserved during the integration process, then, at brief SOAs, subjects ought to respond with the composite pattern. The analysis focused on the types of errors that subjects made when attempting to identify a target in the presence of a backward masker.

Although some previous studies have investigated vibrotactile masking using letters of the alphabet (Craig, 1982, 1983), the large amount of redundancy inherent in such stimulus sets often makes it difficult to assess the perceptual effects of a masking stimulus (Evans \& Craig, 1986; Lupker, 1979). The stimulus set used in the present study had no redundancy. In other words, all of the information contained within any one pattern was necessary to uniquely identify that pattern.

\section{Method}

Subjects. Three subjects were tested. All were sighted, paid employees of the laboratory and had served in related experiments.

Apparatus. Stimuli were presented to the subject's left index fingerpad using the $6 \times 24$ array of stimulators of the Optacon. When activated, each stimulator in the array vibrates at a frequency of approximately $230 \mathrm{~Hz}$. Stimulus generation and presentation was controlled by a PDP-11/34 computer, which also recorded subjects' responses and presented feedback. A more detailed description of the apparatus may be found in Craig (1980).

Stimuli. The target stimuli consisted of patterns that contained one, two, or three lines (Figure 1). A line was generated by turning on either two columns or two rows on the Optacon display. There were four patterns containing one line (Set I), five patterns containing two lines (Set II), and four patterns containing three lines (Set III). Each target pattern also served as a masking stimulus.

Procedure. The subject sat with his/her left index fingerpad resting on the tactile array. In front of the subject was a CRT (cathoderay tube) screen that displayed instructions and presented trial-bytrial feedback. To the right of the subject was a standard computer keyboard used for indicating responses. To eliminate auditory cues from the tactile array, the subject wore headphones through which white noise was presented.

On any trial the particular target and masker to be presented was determined randomly. However, there was one restriction: On 25\% of the trials, the masker was the same as the target pattern. The reason for this was that previous research using letter stimuli had shown that a minimal amount of masking occurs when two identical letters are presented in close temporal succession (Craig \& Evans, 1987). This would be expected if two patterns presented in close temporal succession were integrated into a composite in which the spatial locations of the elements of the target and masker were preserved. In this case, the composite pattern would be identical to the target. To provide sufficient data to analyze these same pairs, the percentage of identical target/masker combinations was increased from $8 \%$ ( 1 in 13 by chance alone) to $25 \%$.

The target and the masker were each presented for $26 \mathrm{msec}$, and the intensity of the target and masker was set at $30 \mathrm{~V}$ to the pins of the display. The temporal separation between the onsets of the target and masker (the SOA) was varied. The masker followed the target pattern at one of seven different SOA values: $26,46,66$, $106,186,346$, and $526 \mathrm{msec}$. Identification performance in the absence of a masker was also measured.
A card in front of the subject illustrated the 13 patterns to be identified. Each pattern was numbered (1 through 13), and the keyboard was numbered similarly. The subject was told that he/she would be presented with two patterns and that he/she was to identify the first pattern and to indicate his/her decision by pressing one of the response buttons on the keyboard. If the decision was correct, the word "correct" appeared on the CRT screen. A wrong decision was followed by the number corresponding to the presented target pattern appearing on the screen. Subjects performed at their own pace and controlled the stimulus presentation by pressing the space bar on the keyboard.

Subjects completed one experimental session per day for a total of 20 sessions. Trials were blocked by SOA, and an experimental session consisted of eight blocks, each consisting of 40 trials. Each session was a complete within-subject replication of the experiment.

\section{Results and Discussion}

Before the results are presented, two issues need to be considered. First, since the question addressed in Experiment 1 was whether, at brief SOAs, subjects would respond with the inclusive composite of the target and masker, the analysis focused on the target/masker combinations for which subjects' identification errors could unambiguously be categorized as "composite" responses or "other" responses. As an example, Target 1 followed by Masker 8 (see Figure 1) fullfills the requirement (the inclusive composite is Pattern 12). Target 5 followed by Masker 6 does not fulfill the requirement since these two patterns share a line, and if subjects responded with the composite pattern (Pattern 10), it would not be clear from this response if this was a result of complete integration of all of the lines in Pattern 5 with some of the lines in Pattern 6, or the reverse. Other target/masker combinations were also excluded from the analysis, since they did not form composites that were available to subjects as responses (e.g., Target 6 followed by Masker 11). As a result of these restrictions, 30 different target/masker combinations were included in the subsequent analysis. ${ }^{1}$

A second issue that needs to be considered is that previous research by Evans and Craig (1986) has shown that the ability to identify a spatial, vibrotactile target pattern in the absence of a masking stimulus depends, in part, on the number of lines contained in the pattern: the more lines, the less likely subjects are to correctly identify the pattern. The present results also showed this. In the absence of a masking stimulus, subjects identified the oneline targets $97 \%$ of the time, the two-line targets $75 \%$ of the time, and the three-line targets $67 \%$ of the time. The importance of this finding is that since a composite of a target and masker will contain more lines than are contained in the target or in the masker, there may be occasions when subjects base their identification decisions on the inclusive composite but then "misidentify" the composite pattern because it is relatively complex. If this is the case, then we should expect the number of composite responses to vary as a function of the complexity of the target and masker patterns that make up the composite. Thus, the 30 different target/masker combinations were partitioned in terms of the number of lines contained in the target and in the masker. There were 10 target/masker 


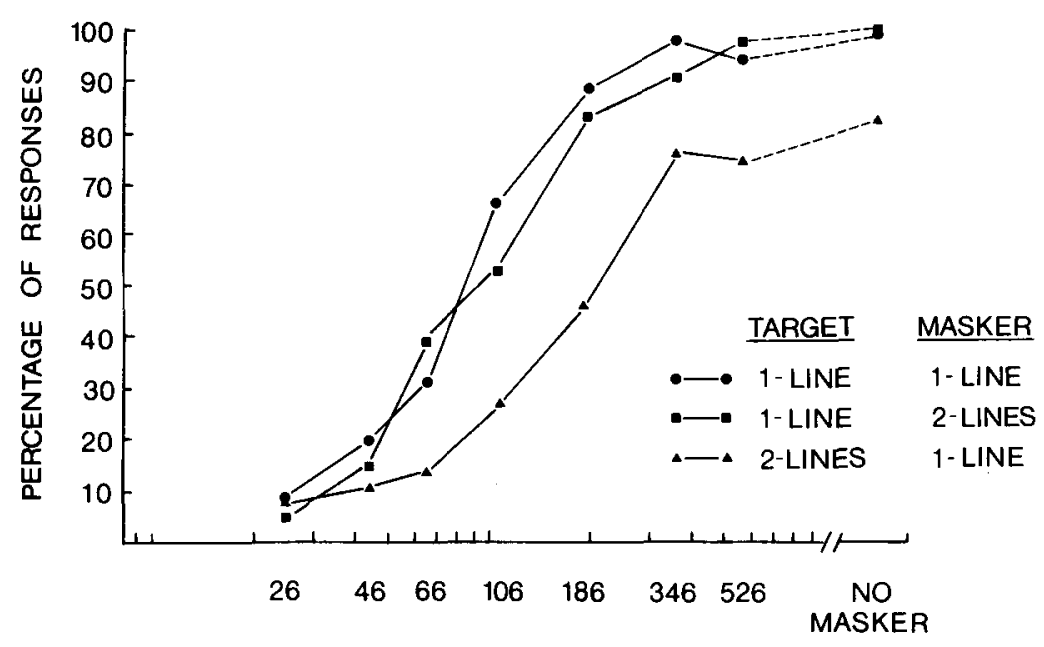

Figure 2. Percentages of target responses (correct identifications) for the three target/masker stimulus sets as a function of SOA (backward-masking condition).

pairs in which both the target and the masker were from Set I, 10 pairs in which the target was from Set I and the masker was from Set II, and 10 pairs in which the target was from Set II and the masker was from Set I.

Figure 2 shows the percentages of target responses (correct identifications) for the three sets of target/masker combinations as a function of SOA. There was an average of 115 observations for each SOA condition. The standard errors of the means ranged from $2 \%$ to $9 \%$ and did not vary systematically with SOA. Also shown, for comparison purposes, is the single-pattern identification performance.

An analysis of variance performed on these data showed a significant effect of target/masker stimulus set $[F(2,4)$ $=13.68, p<.02]$, a significant effect of SOA $[F(7,14)$ $=159.43, p<.001]$, and a significant interaction between target/masker stimulus set and SOA $[F(14,28)=$ $4.20, p<.001]$. Post hoc comparisons (Tukey HSD method) showed that at SOAs of 26 and $46 \mathrm{msec}$ there were no significant differences between the percentages of correct identifications for the three sets of target/masker combinations (critical value $=14.2, p>.05$ ). However, at SOAs greater than $46 \mathrm{msec}$, the one-line target patterns, when they were followed by either a one-line masker or a two-line masker, were identified significantly better than the two-line target patterns ( $p<.01$ in all cases).

As can be seen in Figure 2, performance for each set of target/masker combinations was worse at the briefest SOAs and increased as the SOA was increased. Post hoc comparisons showed a significant amount of interference for the one-line targets at SOAs up to and including $106 \mathrm{msec}$ (critical value $=18.8, p<.01$ ). For the twoline targets, a significant amount of interference was also noted at $186-\mathrm{msec}$ SOA $(p<.01)$. Beyond 106-msec SOA for the one-line targets and beyond 186-msec SOA for the two-line targets, performance in the presence of a masker was not significantly different from that observed in the absence of a masker.
Clearly, at the briefest SOAs, subjects were making many identification errors. What was the nature of these errors? Did subjects for example, err and respond with the inclusive composite of the target and masker? The percentages of composite responses, for each set of target/masker combinations, are shown in Figure 3 as a function of SOA.

An analysis of variance performed on these data showed a significant effect of target/masker stimulus set $[F(2,3)$ $=17.57, p<.02]$, a significant effect of SOA $[F(6,12)$ $=10.67, p<.001]$, and a significant interaction between target/masker stimulus set and SOA $[F(12,24)=7.25$, $p<.001]$. Post hoc comparisons showed that at SOAs of 26,46 , and $66 \mathrm{msec}$, there were significantly more composite responses made when the target and masker both contained one line than when the target contained one line and the masker contained two lines or when the target contained two lines and the masker contained one line (critical value $=12.7, p<.01$ in all cases). In other words, when the target and masker were both relatively simple, subjects often did respond with the inclusive composite. In addition, at SOAs of 26 and $46 \mathrm{msec}$, significantly more composite responses were made when the target contained one line and the masker contained two lines than when the reverse was true $(p<.01)$. When the target contained two lines and the masker contained one line, very few composite responses were made beyond $26 \mathrm{msec}$.

The fact that subjects often did respond, at brief SOAs, with the inclusive composite pattern supports and extends the temporal integration explanation of vibrotactile backward masking proposed by Evans and Craig (1986). When two vibrotactile patterns are presented in close temporal succession, they are integrated into a composite that contains both the target and the masker. Since subjects did often err and respond with the inclusive composite pattern, information about the spatial locations of lines contained in the target and masker patterns must be preserved 


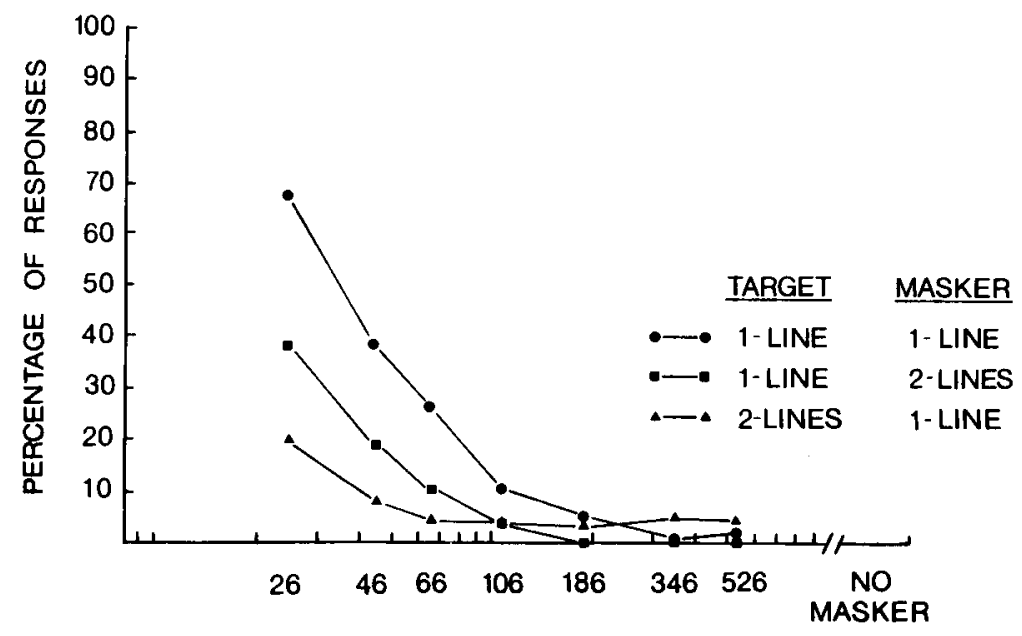

Figure 3. Percentages of composite responses for the three target/masker stimulus sets as a function of SOA (backward-masking condition).

during the integration process. If this is the case, then we should expect to observe very little interference when a target pattern is followed by an identical masker. An analysis of these "same" target/masker pairs showed this to be true. When a target pattern was followed by an identical masker, there was very little interference. The amount of interference, if any, ranged from $1 \%$ to $4 \%$ and did not vary as a function of SOA. If the spatial locations of lines contained in the target and masker patterns are not preserved, we should expect to observe some masking, since the composite representation would be different from either the target or the masker. Craig and Evans (1987), using letter patterns, also showed a minimal amount of masking when two identical letters were presented in close temporal succession.

Why were very few composite responses made when the target or the masker was relatively complex? Assuming that the integration process has a duration of approximately $100 \mathrm{msec}$ irrespective of whether the presented stimulus is simple or complex in spatial detail, it seems plausible to argue that subjects are always basing their identification decisions at brief SOAs on a composite representation containing both the target and masker pattern but that when the composite is relatively complex, they may "misidentify" it. As the results of the singlepattern identification condition show, subjects were better able to identify the targets that contained relatively few lines than they were the targets that contained relatively many lines, and presumably the same would hold true when subjects base their identification decisions on a composite representation.

Even when the target and the masker were relatively simple, the target and the composite response categories did not account for all of the responses that subjects made. For example, at 26-msec SOA, subjects responded with the target pattern $7 \%$ of the time and with the inclusive composite pattern $68 \%$ of the time. This leaves $25 \%$ of their responses, at 26-msec SOA, unaccounted for. Were these responses misidentifications of the target pattern and "misidentifications" of the composite pattern? To answer this question, subjects' responses not accounted for by the target and composite response categories, for each of the three target/masker stimulus sets, were examined. The results of this examination revealed that often, when not responding with the target or with the composite, subjects erred and responded with the masker pattern, the pattern they were instructed to ignore. An analysis of variance performed on these data showed a significant effect of SOA $[F(6,12)=16.63, p<.001]$. There was no effect of target/masker stimulus set $[F(2,4)=0.444, p>$ $.05]$ and no interaction between target/masker stimulus set and SOA $[F(12,24)=1.00, p>.05]$. The percentage of masker responses, averaged across the three target/masker stimulus sets, are shown in Figure 4 as a function of SOA.

As shown by $t$ tests, there were significantly more masker responses made at SOAs of $26,46,66$, and $106 \mathrm{msec}$ than would be expected by chance alone ( $p<.01$ in all cases). Post hoc comparisons showed that significantly more masker responses were made at 46-msec SOA than were made at 26-msec SOA (critical value $=19.4, p<.01$ ). The difference between the percentages of masker responses made at $26 \mathrm{msec}$ and at $66 \mathrm{msec}(19 \%$ vs. $26 \%)$ was not statistically significant $(p>.05)$.

Why, in a backward-masking paradigm, might subjects sometimes err and respond with the masker pattern? One possibility is that in a masking paradigm, the temporally trailing pattern is, at brief SOAs, represented somewhat more strongly in the composite than is the temporally leading pattern. The trailing pattern may be represented more strongly than the leading pattern because it has faded proportionally more than the leading pattern by the time an identification decision is made (the fadingtrace hypothesis). The possibility also exists that the arrival of the trailing pattern actively suppresses the response 


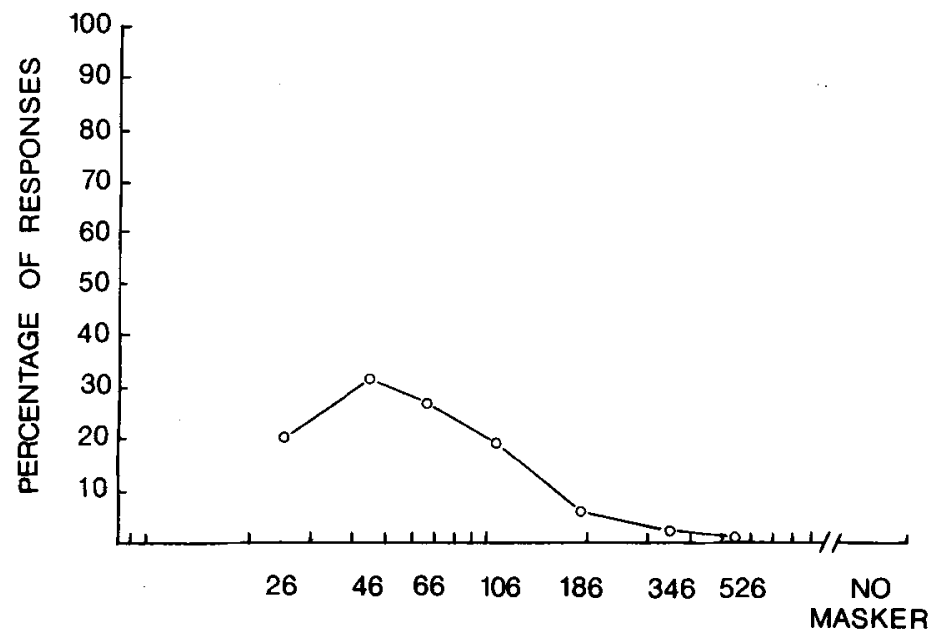

Figure 4. Percentage of masker responses, averaged across the three target/masker stimulus sets, as a function of SOA (backward-masking condition).

of the system to the leading pattern (the suppression hypothesis). The present study does not test between these two hypotheses (see Di Lollo \& Hogben, 1987). Experiment 2 simply tests the idea that the trailing pattern is represented more strongly in the composite than is the leading pattern.

\section{EXPERIMENT 2}

The results of Experiment 1 suggest that a composite representation, consisting of the representations of both the temporally leading and the temporally trailing pattern, is formed when two vibrotactile patterns are presented in close temporal and spatial proximity. However, the relative strength of the representation of each pattern in the composite representation may depend on the temporal position of a pattern. Specifically, in the composite representation, the temporally trailing pattern may be represented more strongly than the temporally leading pattern. Raising the strength of representation by moving a pattern from a leading to a trailing position may cause subjects to respond with the pattern more frequently. Experiment 2 addressed the strength of representation question more directly by varying the intensity of a target pattern. The assumptions were that increasing the intensity of a pattern would lead to an increase in the strength of the representation of that pattern, and that as the strength of the representation of a pattern increased, the more clearly the pattern would be perceived.

A backward-masking paradigm was used. Subjects were presented with the patterns shown in Figure 1. These patterns were used as target stimuli and as backward maskers. The temporal separation between the onsets of the target and masker was varied, and the strength of the representation of the target, the temporally leading pattern, was manipulated by varying the intensity of vibration of the pins making up the lines of the target. This manipulation is analogous to increasing the intensity of a visually presented pattern. Previous research had shown that as the intensity of a vibrotactile pattern is increased, identification performance improves (Craig, 1978). The intensity of the masker, the temporally trailing pattern, was fixed. If subjects sometimes respond with the trailing pattern, the masker, because it is represented more strongly in the composite than is the leading pattern (Experiment 1 ), then increasing the strength of the representation of the leading pattern, the target, should systematically decrease the percentage of masker responses.

\section{Method}

Subjects. Seven subjects were tested. None of them had participated in Experiment 1. All were experienced laboratory subjects and had served in related experiments.

Apparatus and Stimuli. The apparatus and stimuli used in Experiment 1 were used in Experiment 2.

Procedure. The general procedural details were identical to those of Experiment 1. The subjects were presented with two patterns and were instructed to identify the first pattern. The temporal separation between the target and masker was varied. Four SOA values were tested-26, 46, 66, and $106 \mathrm{msec}$. The intensity of the target pattern was varied by varying the voltage to the stimulators of the tactile array. The target patterns were presented at four different intensities-18, 27, 36, and $45 \mathrm{~V}$. The masker intensity was fixed at $27 \mathrm{~V}$. Thus, the target was presented at an intensity below that of the masker, equal to that of the masker, and at two intensities greater than that of the masker. When both the target and masker were presented at the same intensity $(27 \mathrm{~V})$, the situation was similar to that of Experiment 1, except that in Experiment 1 both the target and the masker were presented at $30 \mathrm{~V}$.

Trials were blocked by SOA, and the intensity of the target pattern was varied randomly on a trial-to-trial basis. Each session consisted of eight blocks, and the subjects completed a total of eight sessions.

\section{Results and Discussion}

Figure 5 shows the percentages of masker responses, for each SOA condition, as a function of the intensity of the target pattern. The standard errors of the means ranged from $3 \%$ to $7 \%$. An analysis of variance performed on 


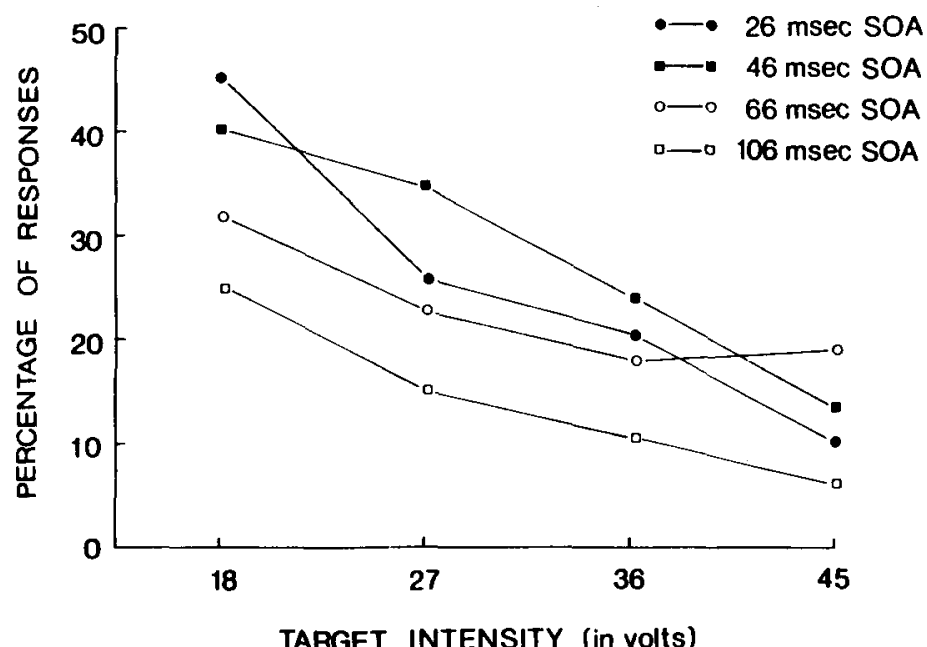

Figure 5. Percentages of masker responses for each SOA condition as a function of the intensity of the target pattern (backward-masking condition).

these data showed a main effect of intensity $[F(3,18)=$ $11.63, p<.001]$ and a main effect of SOA $[F(3,18)=$ $7.91, p<.001]$. There was no interaction between intensity and $\operatorname{SOA}[F(9,54)=1.75, p>.10]$.

Post hoc comparisons (Tukey HSD method) showed that significantly more masker responses were made when the target was presented at an intensity less than that of the masker pattern than when the target was presented at the highest intensity (critical value $=22.2, p<.01$ ). From the lowest target intensity to the highest target intensity, there was a decreasing likelihood for the subjects to respond with the masker pattern. In addition, and as would be expected, as the SOA was increased, the subjects made fewer and fewer errors.

Could it be argued that the decrease in the percentage of masker responses was simply a result of subjects' increasing their percentage of target responses as the intensity of the target pattern was increased? An examination of the percentages of target responses as a function of the intensity of the target pattern in the presence of a masker suggests that this was not the case. At 26-msec SOA, at the lowest target intensity, subjects correctly identified the target patterns $12 \%$ of the time. The percentage of correct target responses increased by $12 \%$ to $24 \%$ at the next highest target intensity. As the intensity of the target was increased further, the percentage of target responses did not increase. However, at 26-msec SOA, and over the same range of target intensities, the percentage of masker responses fell from $46 \%$ to $11 \%$, a decrease of $35 \%$. The same general trend was observed at $46-\mathrm{msec}$ SOA; that is, there was a greater decrease in the percentage of masker responses than there was an increase in the percentage of target responses.

The finding of a decrease in the number of masker responses as the intensity of the target pattern was increased supports the hypothesis that the reason subjects may sometimes respond with the masker pattern in a backward-masking paradigm is because, at brief SOAs, the masker is represented more strongly in the composite representation. Increasing the strength of the representation of the target pattern in the composite representation decreases the relative difference between the strengths of the target and masker in the composite. As the relative difference between the strengths of the representations of the target and masker is reduced in a backward-masking paradigm, subjects are less likely to base their identification decisions on the masker pattern.

Notice that when the target and masker were both presented at the same intensity (as in Experiment 1), there was a tendency for subjects to make more masker responses at 46-msec SOA than at 26-msec SOA. In Experiment 1 , the subjects made significantly more masker responses at 46-msec SOA than at 26-msec SOA. At the present time, I have no explanation of the initial increase in masker responses with increasing SOA.

\section{EXPERIMENT 3}

If, at brief SOAs, subjects base their identification decisions on a composite representation that contains a relatively weak representation of the temporally leading pattern (the target in a backward-masking paradigm) and a relatively strong presentation of the temporally trailing pattern (the masker in a backward-masking paradigm), then this would provide one explanation of the often cited finding of more backward than forward masking at brief SOAs (Bliss et al., 1966; Craig, 1983). In a forwardmasking paradigm, the pattern to be identified is, according to the present proposal, the more strongly represented pattern in the composite representation. If, at brief SOAs, subjects do sometimes base their identification decisions on the more strongly represented pattern in the compo- 
site, then responding with the more strongly represented pattern in a forward-masking paradigm will increase the frequency of correct identifications.

Experiment 3 was essentially identical to Experiment 1. However, instead of instructing subjects to identify the first pattern that was presented (backward masking), they were instructed to identify the second pattern (forward masking). The expectation was that there would be less masking at brief SOAs than in the backward-masking condition (Experiment 1), and that rarely would subjects respond with the masker pattern, because, according to the present proposal, in a forward-masking paradigm the masker will be the less strongly represented pattern in the composite representation.

\section{Method}

Subjects. Five subjects who participated in Experiment 2 were tested.

Apparatus and Stimuli. The apparatus and stimuli used in Experiments 1 and 2 were used in Experiment 3.

Procedure. In general, the procedural details were identical to those of Experiment 1, with the exception that a forward-masking paradigm was used rather than a backward-masking one. The target followed the masker pattern at one of seven different SOA values-26, 46, 66, 106, 186, 346, and $526 \mathrm{msec}$. Subjects completed a total of 10 sessions. Trials were blocked by SOA, and an experimental session consisted of 8 blocks, each consisting of 40 trials. Each session was a complete within-subject replication of the experiment.

\section{Results and Discussion}

The data were analyzed exactly as in Experiment 1. Figure 6 shows the percentages of target responses (correct identifications) for the three sets of target/masker combinations as a function of SOA. There was an average of 96 observations for each SOA condition. The standard errors of the means ranged from $1 \%$ to $10 \%$ and did not vary systematically as a function of SOA. Also shown, for comparison purposes, is the single-pattern identification performance.
Identification performance, as in Experiment 1, was worst at the briefest SOAs and improved as the SOA was increased. The results of an analysis of variance performed on these data showed a main effect of SOA $[F(7,28)=35.10, p<.001]$ and a main effect of target/masker stimulus set $[F(2,8)=14.85, p<.01]$. There was no interaction between target/masker stimulus set and SOA $[F(14,56)=1.58, p>.10]$. Post hoc comparisons (Tukey HSD method) showed that the one-line targets, when they were followed by either a one-line masker or a two-line masker, were identified better, overall, than were the two-line targets (critical value $=20.4, p<.01$ ). Performance in the absence of a masker, for each set of target/masker combinations, was similar to that noted in Experiment 1 (compare Figures 2 and 6).

A comparison of Figures 2 and 6 also shows that there was more backward masking, at brief SOAs, than forward masking. This finding is typical of findings in the literature (Bliss et al., 1966; Craig, 1983). However, although performing better in Experiment 3 (forward masking) than in Experiment 1 (backward masking), subjects still made many identification errors at the briefest SOAs. What was the nature of these errors? For example, did the subjects often err and respond, as in Experiment 1 , with the inclusive composite of the target and masker?

Figure 7 shows the percentages of composite responses, for each of the three target/masker combination sets, as a function of SOA. An analysis of variance performed on these data showed a significant effect of target/masker stimulus set $[F(2,8)=22.09, p<.001]$, a significant effect of SOA $[F(6,24)=15.48, p<.001]$, and an interaction between target/masker stimulus set and SOA $[F(12,48)=5.63, p<.001]$. Post hoc comparisons showed that at SOAs of 26,46 , and $66 \mathrm{msec}$, significantly more composite responses were made when the masker ' pattern contained only one line than when the masker contained two lines (critical value $=14.7, p<.01$ ). In Ex-

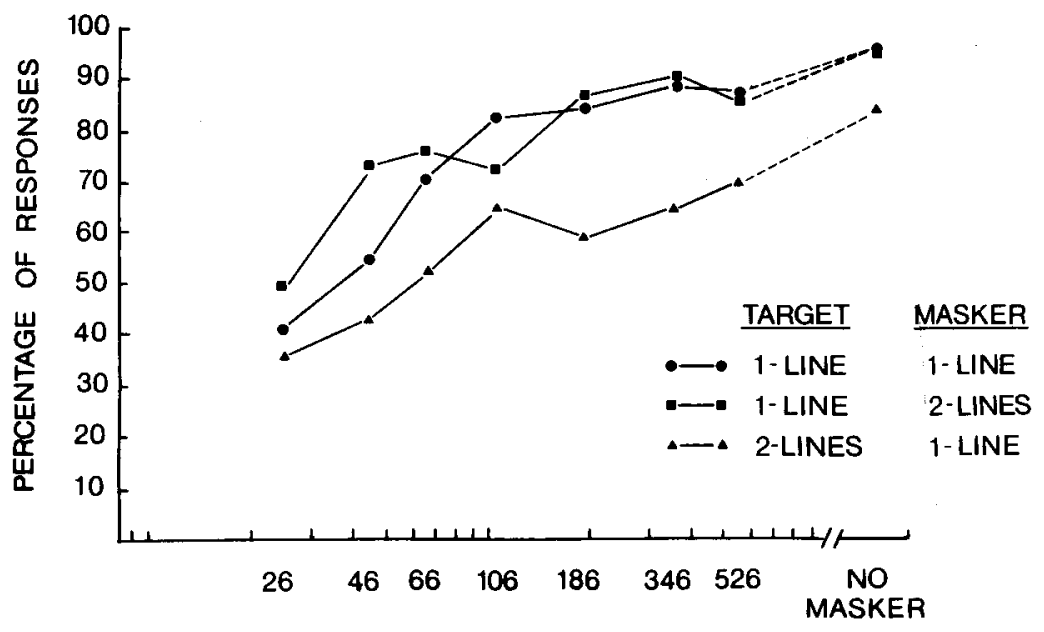

Figure 6. Percentages of target responses (correct identifications) for the three target/masker stimulus sets as a function of SOA (forward-masking condition). 


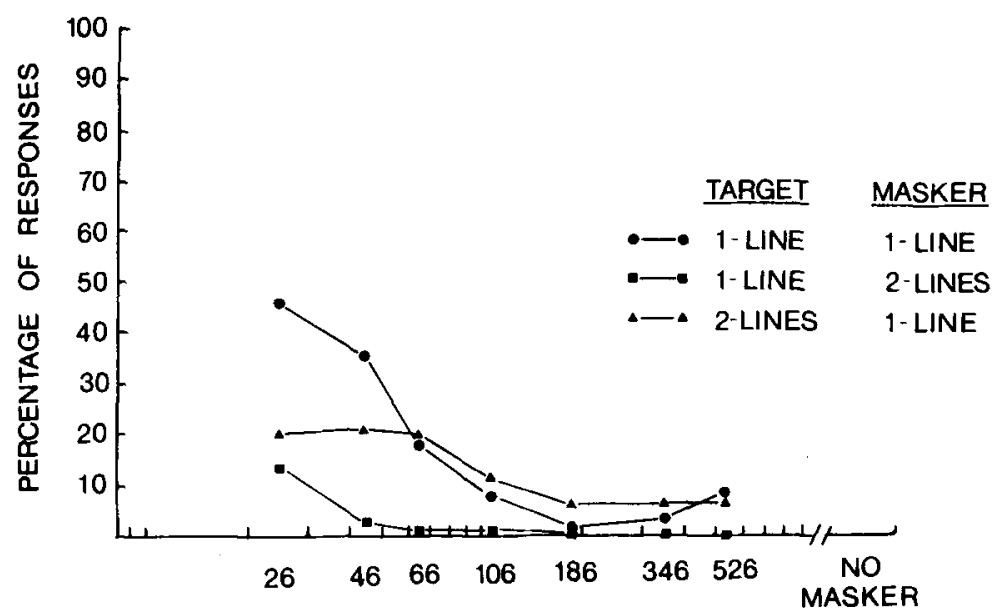

Figure 7. Percentages of composite responses for the three target/masker stimulus sets as a function of SOA (forward-masking condition).

periment 1 , significantly fewer composite responses were made when the target pattern, not the masker, contained two lines. The disparity between these two findings is reconciled when one considers the more general statement that, at brief SOAs, significantly fewer composite responses were made when the temporally leading pattern (the target in a backward-masking paradigm and the masker in a forward-masking paradigm) was relatively complex (when it contained two lines) than when the temporally leading pattern was relatively simple (when it contained one line). As in Experiment 1, the percentage of composite responses decreased overall as the SOA was increased. Beyond 66-msec SOA, very few composite responses were made.

The target and composite response categories did not account for all of the responses made by the subjects. Did the subjects sometimes make an additional type of systematic error at brief SOAs, perhaps responding with the masker pattern as in Experiment 1? If they did, then this would argue against the idea that subjects sometimes respond with the masker pattern in a backward-masking paradigm because it is more strongly represented in the composite representation. According to the present proposal, in a forward-masking paradigm it is the target pattern that is represented more strongly in the composite representation.

Subject responses not accounted for by the target and composite response categories were examined. The results of this examination revealed that subjects did not, as in Experiment 1, often respond with the masker pattern. The percentage of masker responses ranged from $1 \%$ to $11 \%$ for the case when the target contained one line and the masker contained two lines, from $1 \%$ to $7 \%$ for the case when the target contained two lines and the masker contained one line, and from $1 \%$ to $5 \%$ for the case when the target contained one line and the masker contained one line and did not vary systematically as a function of SOA. Masker responses would be expected about $8 \%$ of the time by chance. Thus, in a forward-masking paradigm, the subjects were not systematically erring and responding with the masker pattern. This finding would be expected if the masker pattern, in a forward-masking paradigm, was weakly represented in the composite. Presumably, the responses that subjects made that were not accounted for by the target and composite response categories were misidentifications of the target and "misidentifications" of the composite pattern. No other systematic types of errors were noted.

\section{GENERAL DISCUSSION}

The results of the present study support four conclusions. First, the presentation of a vibrotactile pattern yields an internal representation that persists for some time following the cessation of stimulation. Second, two vibrotactile patterns that were presented in close temporal and spatial proximity are integrated into a composite representation upon which subjects base their identification decisions. Third, information about the spatial locations of target and masker elements is preserved during the integration process. Fourth, the temporally trailing pattern in a masking paradigm is represented more strongly in the composite representation than is the temporally leading pattern.

Conclusions 1, 2, and 3 are based on the finding that subjects often did, at brief SOAs, respond with the composite of the target and masker patterns. A significant number of composite responses was noted at SOAs of 26 , 46 , and $66 \mathrm{msec}$. Since the patterns had presentation durations of $26 \mathrm{msec}$, a representation of the first pattern must persist following the offset of stimulation in order for it to be integrated with a subsequently presented pattern. If information about the spatial locations of lines contained within the two patterns was not preserved during the integration process, we would have expected subjects to respond at brief SOAs with a pattern that contained more lines than were contained in the target, but with one that was not necessarily the inclusive composite pattern. 
The fourth conclusion is based on the finding that subjects sometimes erred and responded with the masker pattern when it followed the target (backward masking), but rarely did so when it preceded the target pattern (forward masking). Increasing the strength of the representation of the temporally leading pattern (thus reducing the disparity between the strengths of the two patterns in the composite representation) led to a systematic decrease in the percentage of trailing-pattern responses (Experiment 2).

That a representation of a vibrotactile pattern persists for some time following its presentation is suggested by a number of findings in addition to those reported here. For example, measurements of the threshold for detecting trains of tactile pulses have shown that the threshold is lower, relative to the threshold for a single pulse, even when the temporal separation between the pulses is as long as $500 \mathrm{msec}$ (Verrillo, 1965). In addition, the results from studies of detection masking suggest that a tactile stimulus yields a representation that persists for several hundred milliseconds (Craig, 1978; Gilson, 1969; Sherrick, 1964). Craig and Evans (1987) have suggested that the reason a forward masker causes more interference than a backward masker at relatively long SOAs is because the persisting representation of the forward masker interferes with the identification of a subsequently presented target pattern.

The present characterization of an internal representation of a vibrotactile pattern that fades with time is similar, in some respects, to the traditional view of sensory store whose contents begin to decay as soon as the inducing stimulus is turned off (Coltheart, 1980). Some investigators have questioned the viability of a concept such as sensory store, and, although my intention is not to enter the debate here (see Coltheart, 1975, 1980; Holding, 1975), some clarifying comments may be useful.

A relatively recent study by Di Lollo (1980) has shown that the traditional storage theory of sensory store is inadequate. Working in the visual domain, Di Lollo showed that strength of visual persistence was inversely related to the duration of the inducing stimulus. The results indicated that the crucial factor in visual temporal integration is the interval between the onsets of two stimuli (SOA), and not, as predicted by the storage theory of persistence, the interval between the offset of the temporally leading stimulus and the onset of the temporally trailing stimulus (interstimulus interval, or ISI). Craig (1983), working in the tactile domain, has also shown that SOA, and not ISI, is the best measure of the interaction between sequentially presented vibrotactile patterns. In the context of the present study, the implication of these results is that the representation of a vibrotactile pattern begins to fade as soon as the pattern has been registered, that is, as soon as the internal representation has formed, and not, as implied by the storage theory of persistence, with the cessation of stimulation.

Presumably, the fading of the representation of a pattern will contribute to the disparity between the strengths of the representations of a target and masker in the com- posite. The question is whether simple fading alone accounts for the disparity (the fading-trace hypothesis) or whether, for example, there is an active process of suppression (the suppression hypothesis). In the visual literature, Di Lollo and Hogben (1987) have argued that the fading representation of a temporally leading pattern does not decay unimpeded in the presence of a subsequently presented pattern. Rather, the duration of visible persistence is reduced if another pattern is presented in close temporal and spatial proximity. In their study, spatial proximity of the trailing pattern was a major determiner of the magnitude of the suppressive effect on the leading pattern. The fact that Loomis and Apkarian-Stielau (1976) have shown that the ability to identify a vibrotactile pattern depends, in part, upon the spatial location of a masking stimulus, is suggestive evidence that a suppressive effect may also occur in vibrotactile masking.

The explanation of vibrotactile masking advanced in this paper is able to account for additional findings in the literature. For example, Bliss et al. (1966) presented subjects with two tactual, alphabetic shapes in rapid succession and required them to identify both of the patterns. At brief SOAs (less than $150 \mathrm{msec}$ ), subjects made many more errors in identifying the first pattern than in identifying the second pattern. In addition, at the briefest SOAs, there was a tendency for subjects, when responding, to reverse the order of input. That subjects made more first-pattern errors than second-pattern errors follows from the present proposal that at brief SOAs subjects are attempting to identify a relatively weakly represented first pattern and a relatively strongly represented second pattern. The tendency of subjects to reverse the order of input when responding parallels the finding in the present study that subjects in the backward-masking condition sometimes responded with the masker pattern. Presumably, Bliss et al.'s (1966) subjects thought that the pattern they were reporting first was the pattern that was presented first when, in fact, it was the second pattern. Likewise, in the present study, subjects presumably thought they were responding with the target pattern when, in fact, it was the masker.

The fact that subjects often did respond in the backwardmasking condition (Experiment 1) with the masker pattern, the pattern they were instructed to ignore, suggests that subjects have little or no knowledge of the temporal order of two stimulus events at brief SOAs. In other words, when subjects are basing their identification decisions on a composite representation containing both target and masker elements, it may be difficult or impossible for them to determine which elements were presented first or second. Such an explanation would also account for the large number of temporal order reversals reported in the study by Bliss et al. (1966). Hirsh and Sherrick (1961) reported that, in trained subjects, the limen for temporal order of transient taps to contralateral fingerpads is approximately $20 \mathrm{msec}$. The results of the present study suggest that the limen for temporal order of two patterns presented to the same fingerpad is greater than $20 \mathrm{msec}$. How subjects do determine which of two sequentially 
presented patterns was presented first or second is an issue of some importance given instructions to subjects in masking paradigms in general to identify the first or the second pattern.

\section{REFERENCES}

Averback, E., Coriell, A. S. (1961). Short-term memory in vision. Bell System Technical Journal, 40, 309-328.

Bliss, J. C., Crane, H. D., Link, S. W., \& Townsend, J. T. (1966). Tactile perception of sequentially presented patterns. Perception \& Psychophysics, 1, 125-130.

Bliss, J. C., Katcher, M. H., Rogers, C. H., \& Shepard, R. P. (1970). Optical-to-tactile image conversion for the blind. IEEE Transactions on Man-Machine Systems, MMS-11, 58-63.

BongarTz, W., \& Scheerer, E. (1976). Two visual stores and two processing operations in tachistoscopic partial report. Quarterly Joumal of Experimental Psychology, 28, 203-219.

Coltheart, M. (1975). Iconic memory: A reply to Professor Holding. Memory \& Cognition, 3, 42-48.

Coltheart, M. (1980). Iconic memory and visible persistence. Perception \& Psychophysics, 27, 183-228.

CraIG, J. C. (1978). Vibrotactile pattern recognition and masking. In G. Gordon (Ed.), Active touch-The mechanism of recognition of objects by manipulation: A multidisciplinary approach (pp. 229-242). Oxford: Pergamon Press.

CRAIG, J. C. (1980). Modes of vibrotactile pattern generation. Joumal of Experimental Psychology: Human Perception \& Performance, 6 , 151-166.

CraIG, J. C. (1982). Vibrotactile masking: A comparison of energy and pattern maskers. Perception \& Psychophysics, 31, 523-529.

Craig, J. C. (1983). The role of onset in the perception of sequentially presented vibrotactile patterns. Perception \& Psychophysics, 34, 421-432.

CRAIG, J. C. (1985). Attending to two fingers: Two hands are better than one. Perception \& Psychophysics, 38, 496-511.

Craig, J. C., \& Evans, P. M. (1987). Vibrotactile masking and the persistence of tactual features. Perception \& Psychophysics, 42, 309-317.

Di LoLlo, V. (1980). Temporal integration in visual memory. Journal of Experimental Psychology: General, 109, 75-97.

Di Lollo, V., \& Hogben, J. H. (1987). Suppression of visible persistence as a function of spatial separation between inducing stimuli. Perception \& Psychophysics, 41, 345-354.
Eriksen, C. W., \& Colluns, J. F. (1967). Some temporal characteristics of visual pattern perception. Journal of Experimental Psychology, 74, 476-484.

Evans, P. M., \& Craig, J. C. (1986). Temporal integration and vibrotactile backward masking. Journal of Experimental Psychology: Human Perception \& Performance, 12, 160-168.

Felsten, G., \& Wasserman, S. S. (1980). Visual masking: Mechanisms and theories. Psychological Bulletin, 88, 329-353.

GILSON, R. D. (1969). Vibrotactile masking: Some spatial and temporal aspects. Perception \& Psychophysics, 5, 176-183.

Hirsh, I. J., \& Sherrick, C. E. (1961). Perceived order in different sense modalities. Journal of Experimental Psychology, 62, 423-432.

Hobgen, J. H., \& Di Lollo, V. (1974). Perceptual integration and perceptual segregation of brief visual stimuli. Vision Research, 14, 1059-1069.

Holding, D. H. (1975). Sensory storage reconsidered. Memory \& Cognition, 3, 31-41.

Loomis, J. M., \& Apkarian-Stielau, P. (1976). A lateral masking effect in tactile and blurred visual letter recognition. Perception \& Psychophysics, 20, 221-226.

LUPKER, J. L. (1979). On the nature of perceptual information during letter perception. Perception \& Psychophysics, 25, 303-312.

Schultz, D. W., ERIKSEN, C. W. (1977). Do noise masks terminate target processing? Memory \& Cognition, 5, 90-96.

SHERrick, C. E. (1964). Effects of double simultaneous stimulation of the skin. American Journal of Psychology, 77, 42-53.

SPENCER, T., \& SHUNTICH, R. (1970). Evidence for an interruption theory of backward masking. Joumal of Experimental Psychology, 85, 198-203.

VERRILlo, R. T. (1965). Temporal summation in vibrotactile sensitivity. Journal of the Acoustical Society of America, 37, 843-864.

\section{NOTE}

1. If subjects were presented with only target/masker combinations that formed composite patterns that were members of the stimulus set, this would have meant that none of the patterns containing three lines could have been presented as targets or maskers. Because this could introduce response biases, all 169 different target/masker combinations were presented.

(Manuscript received April 3, 1987; revision accepted for publication June 30,1987 .) 